Antimatter and Gravity (WAG 2013)

International Journal of Modern Physics: Conference Series

Vol. 30 (2014) 1460255 (9 pages)

(C) The Author

DOI: $10.1142 / \mathrm{S} 2010194514602555$

\title{
Antimatter in the universe and the PAMELA/FERMI/AMS anomaly
}

\author{
G. K. Skinner \\ Max-Planck-Institut für Extraterrestriche Physik \\ 85748 Garching, Germany \\ gerald.k.skinner@gmail.com
}

Received 2 January 2014

Revised 8 March 2014

Published 7 May 2014

\begin{abstract}
Evidence for the existence of antimatter in the Galaxy and in the cosmos as a whole is reviewed and possible explanations that have been proposed for its origin discussed.
\end{abstract}

Keywords: Antimatter; positrons; astrophysics; galaxy.

PACS Numbers: 26.30.Jk, 36.10, 95.85.-e, 96.50.S-, 95.85.Pw, 95.85.Ry

\section{Introduction}

Although there is no indication that any of it is primordial, a significant quantity of antimatter exists in our Galaxy. The evidence for this comes from observations of gamma-rays and of particles. Telescopes on high altitude balloons and spacecraft detect the characteristic gamma-ray line at $511 \mathrm{keV}$ from the annihilation of positrons with electrons. Less direct evidence that processes involving antiprotons are taking place is provided by observations of high energy gamma-rays. Particle detectors, also operating above the atmosphere, find positrons and antiprotons among the cosmic ray particles of energies such that are able to penetrate the solar system.

We review here the current state of knowledge of the galactic antimatter and its origins and then briefly consider the possibility of antimatter elsewhere in the cosmos.

This is an Open Access article published by World Scientific Publishing Company. It is distributed under the terms of the Creative Commons Attribution 3.0 (CC-BY) License. Further distribution of this work is permitted, provided the original work is properly cited. 


\section{Antimatter in Cosmic Rays}

Cosmic rays (CRs) consist of electrons, and of protons and heavier nuclei in proportions that can be understood in terms of the elemental abundances typical of stars and of the interstellar medium (ISM), modified by spallation when CRs interact with the ISM. The observed abundances, particularly the $\mathrm{B}$ to $\mathrm{C}$ ratio, provide insight into how much interstellar material a typical comic ray particle encounters during the time it is in interstellar space. Of interest here is the fact that antimatter in the form of both positrons and antiprotons is observed among the comic ray particles arriving in the vicinity of the earth.

The energy spectrum of cosmic rays drops rapidly with energy — roughly as $E^{-3}$, but with subtle changes in slope - up to more than $10^{20} \mathrm{eV} /$ nucleon. The upper limit is probably that beyond which galactic magnetic fields can confine particles. Turbulent magnetic fields carried by the solar wind prevent cosmic rays below about $10^{8} \mathrm{eV} /$ nucleon reaching the environment of the earth and the particle fluxes observed at lower energies are mainly from the sun.

\subsection{Antiprotons}

The spectrum of the antiprotons that are present in cosmic rays is such that the fraction $\phi(\bar{p}) /(\phi(p)+\phi(\bar{p}))$ rises to $\sim 2 \times 10^{-4}$ at energies above $\sim 1 \mathrm{GeV}$. The best data currently available are from an instrument, the Payload for Antimatter Matter Exploration and Light-nuclei Astrophysics (PAMELA), on a Russian spacecraft launched in June 2006 and are shown in Fig. 1. They are consistent with the production as a result of interactions of cosmic rays with protons and heavier nuclei in the ISM expected from modelling that accounts for the observed flux of matter particles.

\subsection{Cosmic ray positrons - Observations}

Cosmic ray positrons on the other hand present a more complex picture. Like antiprotons, they are expected to be produced as a result of cosmic ray interactions

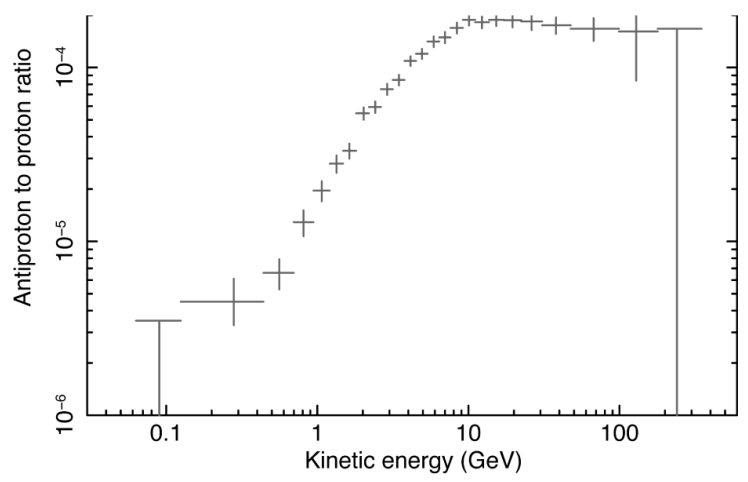

Fig. 1. The antiproton to proton ratio from the PAMELA instrument (data from Ref. 1). 


$\begin{aligned} p p \rightarrow \quad & +X \\ p p \rightarrow K & +X \\ & K^{ \pm} \rightarrow \mu^{ \pm} \nu_{\mu} \\ & K^{ \pm} \rightarrow \pi^{0} \pi^{ \pm}\end{aligned}$

$\begin{aligned} \pi^{0} & \rightarrow \gamma \gamma \\ \pi^{0} & \rightarrow \gamma e^{-} e^{+} \\ \pi^{ \pm} & \rightarrow \mu^{ \pm} \stackrel{(-)}{\nu}_{\mu} \\ \pi^{+} & \rightarrow \bar{\nu}_{\mu} \nu_{e} e^{+}\end{aligned}$

Fig. 2. Some of the interactions of cosmic rays with the interstellar medium relevant to the production of antimatter particles. Here $p$ may represent any nucleus, not just the proton of a Hydrogen atom and $X$ indicates other products that may include antiprotons.

in the ISM. Examples of the processes involved are shown in Fig. 2. Although some early measurements of the positron fraction $\phi\left(e^{+}\right) /\left[\phi\left(e^{-}\right)+\phi\left(e^{+}\right)\right]$with instruments on stratospheric balloons were consistent with expectations based on modelling, there were persistent, mutually discrepant, reports of a higher than expected fraction of positrons at energies greater than about $10 \mathrm{GeV}$ (Fig. 3a).

Results from the PAMELA experiment showed that there is indeed an excess in the positron fraction over expectation in the energy range 10-200 GeV. This unexpected 'Pamela excess' was one of the reasons that efforts were made to install the AMS-02 instrument on the International Space Station, despite an earlier cancellation of the project. The AMS-2 results provide a clear confirmation of the PAMELA findings (Fig. 3b).
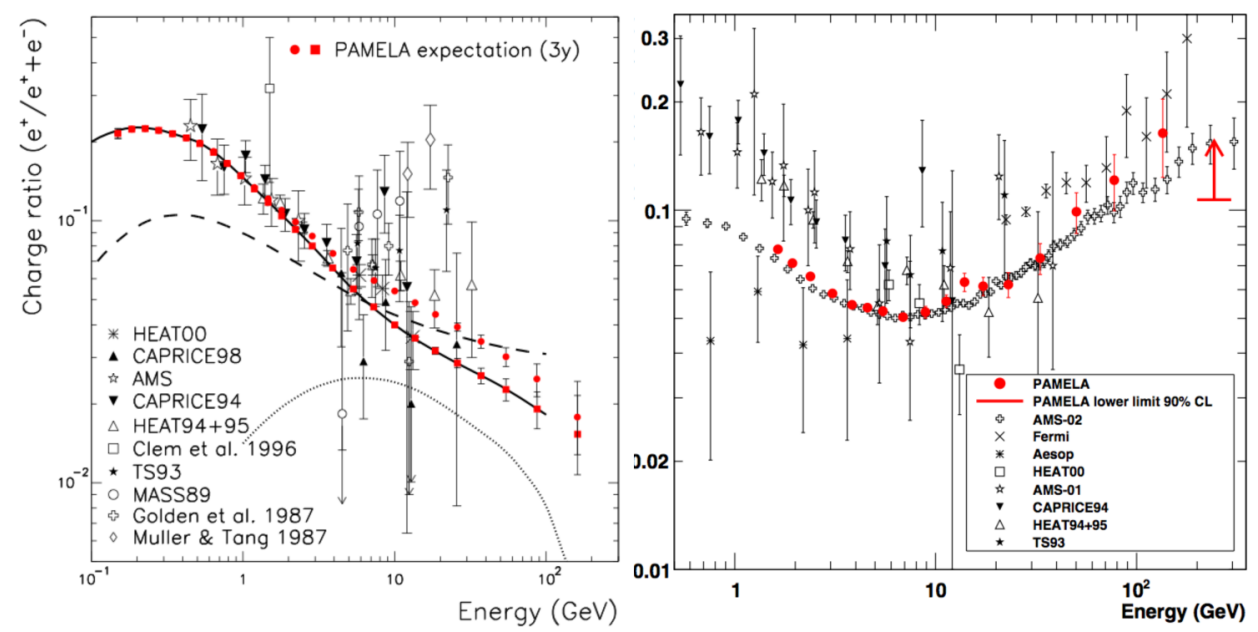

Fig. 3. (Color online) (a, left): Early measurements of the positron fraction in cosmic rays. The red points and thick continuous line indicate what was expected before launch to be seen with PAMELA (circles assume pure secondary positrons, squares include a hypothetical additional primary component). Figure from Ref. 6. (b, right): Observations of the fraction from PAMELA (filled red circles) and AMS-02 (open circles). Figure from Ref. 7. The differences at $\lesssim 1 \mathrm{GeV}$ are thought to be due to varying modulation by interplanetary magnetic fields that change on 22 year cycle and that affect electrons and positrons differently. 
Although having no magnetic spectrometer, the LAT instrument on the FERMI spacecraft has provided further confirmation of the high energy positron excess. Its stacks of tracking detectors, designed to detect pair-production interactions of gamma-rays in the energy range $20 \mathrm{MeV}$ to $300 \mathrm{GeV}$, form an efficient cosmic ray detector. Ackermann et $a .^{2}{ }^{2}$ were able to use the LAT in conjunction with the changing terrestrial magnetic field as the satellite orbits the Earth to further verify the Pamela finding.

Electrons and positrons in the $\sim 100 \mathrm{GeV}$ energy range can be detected at ground level with telescopes designed to detect gamma-rays through the Cherenkov radiation from the showers they produce in the Earth's atmosphere. While showers due to hadrons can be recognised, those produced by gamma-rays can only be separated from showers due to electrons or positrons by the fact that the former come from localised regions on the sky. The $e^{-}+e^{+}$'background' in data from both the H.E.S.S. ${ }^{3}$ and MAGIC ${ }^{4}$ telescopes have been used to extend measurements of the total lepton flux to energies higher than observable with space instruments. An excess is seen to be present, continuing with an $E^{-3}$ spectrum to $\sim 1 \mathrm{TeV}$ where a cut-off sets in. In the future it may become possible to distinguish electrons from positrons with MAGIC though the difference in the position of the shadow of the moon as a result of the geomagnetic field..$^{5}$

\subsection{Cosmic ray positrons - Origins}

The excess in positron fraction in the $\sim 0.1-1 \mathrm{TeV}$ range and in the total lepton spectrum at those energies are consistent with, and suggestive of, an additional component with electrons and positrons in equal numbers. TeV electrons and positrons in interstellar space lose energy quickly through synchrotron radiation and through inverse Compton scattering on Cosmic Microwave Background (CMB) photons. Thus they can travel only relatively short distances and must come from sources within 1-2 kpc (less than $20 \%$ of the distance of the sun from the galactic centre).

One possible origin for the excess is linked to pulsars. The electric field resulting from the strong (up to $\sim 10^{13} \mathrm{G}$ ) rapidly rotating (e.g. $0.1-10^{3} \mathrm{~Hz}$ ) magnetic fields near the poles of the neutron stars in pulsars can give rise to potential differences of up to $10^{16} \mathrm{~V}$. Electrons accelerated by this field and streaming along the magnetic field lines will produce curvature radiation in the form of gamma-rays which then lead to $e^{ \pm}$pair production, either through photon-splitting in the magnetic field or as a result of $\gamma \gamma$ interactions. The particles in the resulting cascade may, or may not, escape the pulsar environment. The pulsar will have been born during a 'core collapse supernova' - the collapse of a massive star. So it will lie initially well within the ejecta from the explosion and by the wind emitted by the star in the late stages of evolution leading up to the collapse, through which the supernova blast wave is propagating outward. For discussion of some of the associated issues see Ref. 8). 
If one or more pulsars are responsible then the most likely candidates are relatively young ones in which the spin is still rapid. The pulsars must in addition be close otherwise there will not have been time since their birth for the $e^{ \pm}$to diffuse through the ISM and reach us.. Among prime suspects are Geminga (age $3.7 \times 10^{5} \mathrm{y}$; period $237 \mathrm{~ms}$ ) and the Monogem pulsar B0656 +14 (1.1 × 10 5 y; $385 \mathrm{~ms})$. Because of beaming, relevant systems may be difficult to detect in the radio band (Geminga is radio quiet and only detected in $\mathrm{X} / \gamma$-rays). The Fermi LAT has now detected high energy gamma-rays from 30 young, radio quiet pulsars. ${ }^{9}$

One of the reasons that the announcement of the positron excess seen with PAMELA attracted so much attention is the possibility that the positrons are the result of the annihilation or decay of dark matter (DM) particles. Of the 1174 citations of the announcement paper ${ }^{10}$ listed in the ADS database, more than half include the word 'dark' in the title. The extent of the literature reflects not just the degree of interest but also the range of possibilities. Dozens of different hypothetical DM particles over a wide range of masses have been suggested to be responsible, either through decay of single long-lifetime particles or from DM-DM interactions. Problems exist in reconciling the implied decay times or cross-sections with theories and modelling that accounts for the freeze-out and condensation of the galactic DM. It has also to be assumed that the active channels are leptonic ones where baryonic channels would in many cases seem more natural. Nevertheless, the possibility that in the Pamela excess we are at last seeing the long sought after non-gravitational signature of dark matter is intriguing.

\section{Gamma-Rays}

The second route through which we learn of antimatter at large in the Galaxy is through observations with gamma-ray telescopes.

Detection of high energy gamma-rays with from two supernova remnants (IC433 and $\mathrm{W} 44^{11}$ ) with a spectrum characteristic of $\pi^{0}$ decay confirms that the processes indicated in Fig. 2 are indeed taking place in the sites where cosmic rays are thought to be accelerated.

The main information on antimatter in the Galaxy comes, however, from observations of the $511 \mathrm{keV}$ electron-positron annihilation line. Pranzos ${ }^{12}$ provides an extensive review of the observations and their implications and here we only outline the key issues and offer a few comments based on recent results.

\subsection{Positron annihilation - Observations}

The $511 \mathrm{keV}$ gamma-ray line was detected coming from a region around the galactic centre with a NaI detector on a stratospheric balloon in $1971^{13}$ and unambiguously identified with a Ge spectrometer on another a few years later. ${ }^{14}$ Since then there have been numerous observations of the emission but the origin of the positrons remains uncertain. The line itself is narrow, but resolved with Ge detectors. It is accompanied by a continuum at energies $<511 \mathrm{keV}$ characteristic of the 3 -photon 
annihilation of positrons that have formed ortho-positronium. In fact, given that for every 3 ortho-positronium atoms formed there will be one para-positronium one that will decay producing two photons in the $511 \mathrm{keV}$ line, the observations are consistent with all $(95 \pm 5 \%)$ of annihilations taking place via the formation of positronium.

Consideration of the positronium fraction and studies of the line width and shape ${ }^{15,16}$ indicate that the annihilations are of positrons of energies less than $\sim 100 \mathrm{eV}$, taking place in a mixture of phases of the ISM referred to as warm neutral $\left(\mathrm{T}=10^{3}-10^{4} \mathrm{~K}\right)$ and warm ionised $(\mathrm{T} \sim 8000 \mathrm{~K})$.

Mapping of the $511 \mathrm{keV}$ emission shows a very strong concentration in an extended region (the 'bulge') around the galactic centre, with some flux coming from the plane of the Galaxy (Fig. 4). Estimates of the flux from the disk depend on its extent in galactic longitude and latitude, which are poorly constrained. But the disk probably accounts for less than half of the total emission. There are persistent indications of an asymmetry in the distribution, with more flux from negative galactic longitudes. Although this was initially reported ${ }^{17}$ as an asymmetry in the disk emission, it is currently thought more likely to be that the centroid of the bulge is offset by $\sim 1^{\circ}$ in that direction. ${ }^{18,19}$
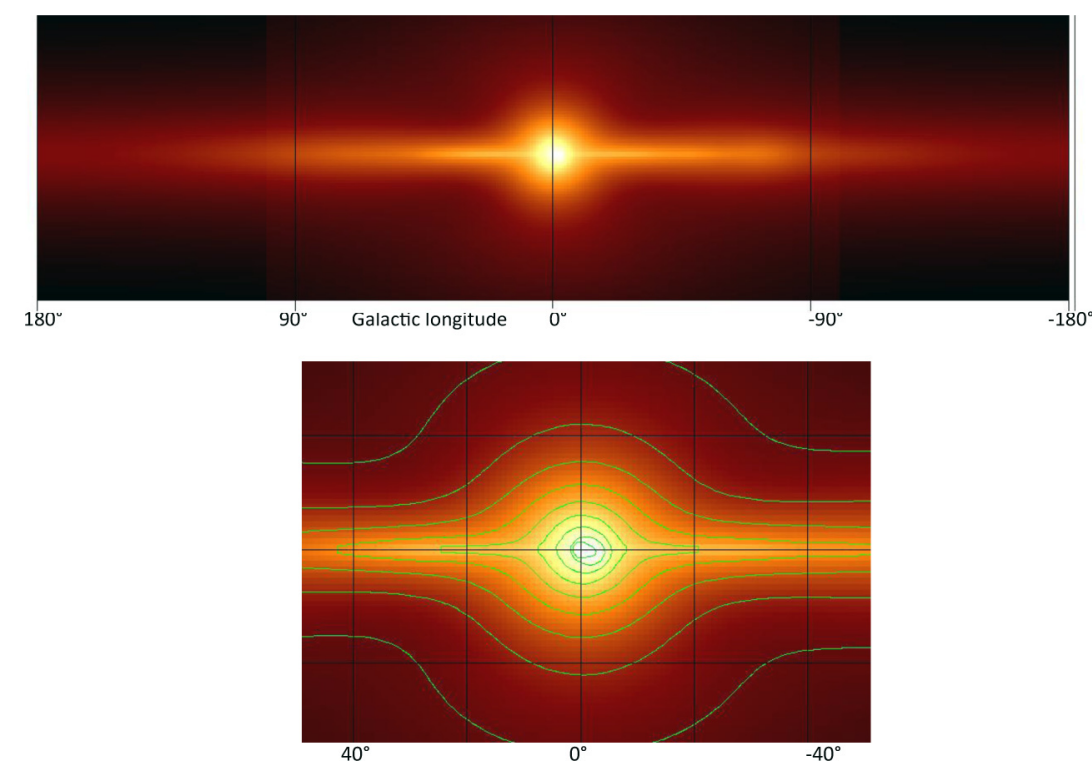

Fig. 4. (Color online) A model of the sky distribution of $511 \mathrm{keV}$ gamma-ray line emission fitted to 10 years of INTEGRAL/SPI data. The galactic centre is in the middle and the galactic plane horizontal. Similar models with different forms for the disc emission provide comparable good fits but all fitted models tend to show the slight offset of the bulge to negative longitudes that is visible in the zoomed image below. 


\subsection{Origin of the annihilating positrons}

Unfortunately knowledge of the environment and locations of positron annihilations provides only limited evidence about where they were created. A large number of sites and mechanisms have been proposed for their origin (see Refs. 12, 18 for some lists).

Two arguments indicate that most of the positrons seen annihilating at low energies are not directly linked to the high energy ones seen in cosmic rays. One is simply based on numbers. Assuming a steady population of positrons in the Galaxy, the observed gamma ray emission implies the creation of a few times $10^{43}$ positrons per second. If modelling of the cosmic ray distribution in the Galaxy is anything like correct and if the positron flux near the Earth is representative of what is going on elsewhere in the Galaxy, then the number of comic ray positrons is insufficient by one or more orders of magnitude. There are several caveats in this statement, but there is a second consideration that argues against any origins in which the initial positron energy exceeds a few $\mathrm{MeV}^{20}$ argue that for higher initial energies a fraction of the positrons would annihilate in flight and the Doppler-shifted annihilation photons produce an excess in the MeV band emission that is not observed.

One mechanism that must produce positrons with initial energies of $\sim \mathrm{MeV}$ is the decay of radioactive isotopes known to be produced in nova and supernova explosions. A major question is whether the decays take place in an environment such that the positrons can escape into the ISM. In some cases escape is very probable but the likely positron production insufficient. For example, the $\sim 10^{6} \mathrm{y}$ lifetime of the ${ }^{26} \mathrm{Al}$ nuclei produced in massive stars and in supernovae allows ample time for them to escape into the ISM before they decay producing a positron in $82 \%$ of cases. Observations of the $1809 \mathrm{keV}$ gamma-ray line from the resulting ${ }^{26} \mathrm{Mg}^{*}$ provides a good measure of the rate of production of positrons by this route and it is more than an order of magnitude below that required. A similar argument applies to ${ }^{44} \mathrm{Ti}$. On the other hand, positrons from the ${ }^{56} \mathrm{Ni} \rightarrow{ }^{56} \mathrm{Co}$ decay chain are produced only a few months after a supernova and probably only a small fraction escape into the ISM, perhaps $1-15 \%$. Estimates of the rate of production of ${ }^{56} \mathrm{Co}$ are also very uncertain, depending on a observations of few atypical examples. Given the flexible constraints, by making optimistic uncertainties it would probably be possible to account for the positron production rate inferred from the total observed gamma-ray flux as due to radioactive decays. However the stellar populations that give rise to the radioactive isotopes are preferentially distributed in the disk of the Galaxy, not in the bulge where most of the annihilations seem to occur.

Attempts have been made to explain how positrons produced in the disk might preferentially find their way to the central regions to annihilate there, either as the result of magnetic fields, ${ }^{21}$ or by travelling large distances through regions of hot ionised ISM, where slowing and recombination are inefficient. ${ }^{22}$ These suggestions rely on rather contrived assumptions and a more natural explanation is that, while 
radioactivity very likely accounts for the positrons that annihilate in the disk, a separate origin is needed to explain the bulge emission.

There have been numerous suggestions that the positrons responsible for the $511 \mathrm{keV}$ line, or at least most of those from the bulge, come from DM. If one accepts the argument that these positrons must have been created with energies no greater than $\sim \mathrm{MeV}$, the only possibilities are then that they come from the annihilation of 'light' DM particles with mass-energy in this range or that they are produced by the decay of an excited state. In either case comparison of the form of the $511 \mathrm{keV}$ emission from the bulge with model DM profiles implies that a 2-body process, proportional to density squared, is involved.

\section{Beyond the Galaxy: Anti-Stars, Anti-Galaxies, Anti-Clusters}

In his 1933 Nobel lecture, after predicting the existence of antiprotons, Dirac said ${ }^{23}$

We must regard it rather as an accident that the Earth (and presumably the whole solar system), contains a preponderance of negative electrons and positive protons. It is quite possible that for some of the stars it is the other way about, these stars being built up mainly of positrons and negative protons. In fact, there may be half the stars of each kind. The two kinds of stars would both show exactly the same spectra, and there would be no way of distinguishing them by present astronomical methods.

The existence of anti-stars seems to be excluded because the inevitable annihilations when stellar winds or supernova ejecta from matter and anti-matter regions interact could not be missed. Even galaxies and anti-galaxies in the same cluster would make themselves apparent because both would be in a common intergalactic gas. But the possibility of anti-matter domains on the scale of clusters of galaxies or larger is harder to dismiss.

\section{References}

1. O. Adriani and 67 others, Soviet Journal of Experimental and Theoretical Physics Letters 96, 621 (2013).

2. M. Ackermann and 159 others, Physical Review Letters 108, 011103 (2012).

3. F. Aharonian and 170 others, Astron. ES Astrophys. 508, 561 (2009).

4. D. Borla Tridon, International Cosmic Ray Conference 6, 43 (2011).

5. P. Colin, International Cosmic Ray Conference 6, 189 (2011).

6. P. Picozza and 59 others, Astroparticle Physics 27, 296 (2007).

7. O. Adriani and 64 others, Physical Review Letters 111, 081102 (2013).

8. P. Blasi and E. Amato, Positrons from pulsar winds, in High-Energy Emission from Pulsars and their Systems, eds. D. F. Torres and N. Rea (2011).

9. A. A. Abdo and 210 others, Astrophys. J. Suppl. 208, 17 (2013).

10. O. Adriani and 50 others, Nature 458, 607 (2009).

11. M. Ackermann and 168 others, Science 339, 807 (2013). 
12. N. Prantzos, C. Boehm, A. M. Bykov, R. Diehl, K. Ferrière, N. Guessoum, P. Jean, J. Knoedlseder, A. Marcowith, I. V. Moskalenko, A. Strong and G. Weidenspointner, Reviews of Modern Physics 83, 1001 (2011).

13. W. N. Johnson, III and R. C. Haymes, Astrophys. J. 184, 103 (1973).

14. M. Leventhal, C. J. MacCallum and P. D. Stang, Astrophys. J. 225, L11 (1978).

15. E. Churazov, R. Sunyaev, S. Sazonov, M. Revnivtsev and D. Varshalovich, Mon. Not. R. Astr. Soc. 357, 1377 (2005).

16. P. Jean, J. Knödlseder, W. Gillard, N. Guessoum, K. Ferrière, A. Marcowith, V. Lonjou and J. P. Roques, Astron. \& Astrophys. 445, 579 (2006).

17. G. Weidenspointner, G. Skinner, P. Jean, J. Knödlseder, P. von Ballmoos, G. Bignami, R. Diehl, A. W. Strong, B. Cordier, S. Schanne and C. Winkler, Nature 451, 159 (2008).

18. G. Skinner, P. Jean, J. Knödlseder, P. Martin, P. von Ballmoos and G. Weidenspointer, The Galactic positron annihilation radiation: an update based on recent SPI observations, in Eighth Integral Workshop. The Restless Gamma-ray Universe (INTEGRAL 2010), (2010).

19. L. Bouchet, J. P. Roques and E. Jourdain, Astrophys. J. 720, 1772 (2010).

20. P. Sizun, M. Cassé and S. Schanne, Phys. Rev. D 74, 063514 (2006).

21. N. Prantzos, Astron. ES Astrophys. 449, 869 (2006).

22. J. C. Higdon, R. E. Lingenfelter and R. E. Rothschild, Astrophys. J. 698, 350 (2009).

23. P. A. M. Dirac, Theory of electrons and positrons (1933), http://www.nobelprize.org/ nobel_prizes/physics/laureates/1933/dirac-lecture.html. 